\title{
BEC-BCS crossover in a cold and magnetized two color NJL model
}

\author{
Dyana C. Duarte, ${ }^{1}$ P. G. Allen, ${ }^{2}$ R. L. S. Farias, ${ }^{1,3}$ Pedro H. A. Manso, ${ }^{4,5}$ Rudnei O. Ramos, ${ }^{4}$ and N. N. Scoccola ${ }^{2,6,7}$ \\ ${ }^{1}$ Departamento de Física, Universidade Federal de Santa Maria, 97105-900 Santa Maria, RS, Brazil \\ ${ }^{2}$ Department of Theoretical Physics, Comisión Nacional de Energía Atómica, Av. Libertador 8250, \\ 1429 Buenos Aires, Argentina \\ ${ }^{3}$ Department of Physics, Kent State University, Kent, Ohio 44242, United States \\ ${ }^{4}$ Departamento de Física Teórica, Universidade do Estado do Rio de Janeiro, \\ 20550-013 Rio de Janeiro, RJ, Brazil \\ ${ }^{5}$ Centro Federal de Educação Tecnológica Celso Suckow da Fonseca, Campus Maria da Graça, \\ Rua Miguel Ângelo 96, 20785-223 Rio de Janeiro, RJ, Brazil \\ ${ }^{6}$ Department of Theoretical Physics, Comisión Nacional de Energía Atómica, CONICET, \\ Rivadavia 1917, 1033 Buenos Aires, Argentina \\ ${ }^{7}$ Universidad Favaloro, Solís 453, 1078 Buenos Aires, Argentina
}

(Received 13 October 2015; published 22 January 2016)

\begin{abstract}
The BEC-BCS crossover for a Nambu-Jona-Lasinio (NJL) model with diquark interactions is studied in the presence of an external magnetic field. Particular attention is paid to different regularization schemes used in the literature. A thorough comparison of results is performed for the case of a cold and magnetized two-color NJL model. According to our results, the critical chemical potential for the BEC transition exhibits a clear inverse magnetic catalysis effect for magnetic fields in the range $1 \lesssim e B / m_{\pi}^{2} \lesssim 20$. As for the BEC-BCS crossover, the corresponding critical chemical potential is very weakly sensitive to magnetic fields up to $e B \sim 9 m_{\pi}^{2}$, showing a much smaller inverse magnetic catalysis as compared to the BEC transition, and displays a strong magnetic catalysis from this point on.
\end{abstract}

DOI: 10.1103/PhysRevD.93.025017

\section{INTRODUCTION}

Although a considerable amount of theoretical and experimental work has been devoted to the subject, the phase diagram of quantum chromodynamics (QCD) still remains poorly understood. From the theoretical point of view, one of the main reasons for this state of affairs is that the $a b$ initio lattice QCD approach has difficulties dealing with the region of medium/low temperatures and moderately high densities, owing to the so-called "sign problem" $[1,2]$. Thus, most of the present knowledge about the strongly interacting matter phase diagram arises from the study of effective models [3]. This is because effective models offer the possibility of obtaining predictions for the transition features at regions that are not accessible through lattice techniques. In this context, in the last years several works have considered that, at low temperatures, the transition between the chirally broken phase at low densities and the color superconducting phase at large densities proceeds in a smooth way instead of being a strong first-order transition, as is more commonly believed. Interestingly, this opens up the possibility that, as density increases, quark matter undergoes a crossover between a regime where diquark pairs form difermion molecules in Bose-Einstein condensation (BEC) and a weakly coupled Bardeen-Cooper-Schrieffer (BCS) superfluid regime [4]. One of the models where this kind of phenomena has been more actively investigated is the well-known NambuJona-Lasinio (NJL) model [5], where gluon-mediated interactions are replaced by effective local quark-quark interactions.

The actual existence of a BEC-BCS crossover in the three-color $\left(N_{c}=3\right)$ NJL model requires, however, a quite strong quark pairing interaction. Let us recall that the application of the Fierz transformations to the effective onegluon-exchange (OGE) interaction leads to $r \equiv G_{D} / G_{S}=$ $3 / 4$, where $G_{S}$ and $G_{D}$ are the scalar quark-antiquark and the diquark coupling constants, respectively. This value is usually taken as a reference in most model calculations [6]. On the other hand, the existence of a BEC-BCS crossover at finite baryon chemical potential requires $r \gtrsim 1[7,8]$. It has been argued, however, that in the flavor SU(3) NJL model the axial anomaly might induce a BEC-BCS crossover for more conventional values of $r[9,10]$. Another possibility has been proposed in Ref. [11]. Contrary to what happens for $N_{c}=3$, the situation for the $N_{c}=2 \mathrm{NJL}$ model is quite clear. In this case, the Fierz transformation of the OGE interaction leads to $r=1$, as shown in Ref. [12]. For this value of $r$, the authors in Ref. [7] found that as the quark chemical potential $\mu$ increases, there is first, at $\mu=m_{\pi} / 2$, a second-order transition from the chirally broken phase to the BEC phase, followed by the BECBCS crossover at a somewhat larger chemical potential. This result was confirmed in Refs. $[13,14]$.

Despite the fact the two-color NJL model might only share some qualitative similarities with real three-color QCD, it is still a valuable model for studying in general. 
In fact, studies with two-color QCD-like models, in particular in the context of the NJL model, have been quite popular (for a recent review, see, e.g., Ref. [15] and references therein). Because of the different gauge group of two-color QCD (which has only pseudoreal, or real representations) as compared to the three-color case, the fermion determinant in the former remains real for nonvanishing chemical potentials (for baryons or quarks). Hence, the model does not suffer from the fermion-sign problem that plagues the three-color QCD and its phase diagram can be studied through lattice Monte Carlo simulations [16-18]. Besides, the two-color NJL model can be seen as a relativistic analogue of the low-energy nonrelativistic BCS-BEC crossover in condensed matter fermionic systems. Given the above properties, we hope to learn some of the related physics associated with diquark condensation in real QCD by using this simpler model and the generic properties connected to a BEC-BCS transition in fermionic systems in general.

In the present work, we will be mainly interested in the role played by a magnetic field in the BEC-BCS transition problem. This is mostly motivated by the realization that strong magnetic fields may be produced in several physically relevant situations. For example, the compact stellar objects believed to be the source of intense $\gamma$ and $x$ rays, the magnetars, are expected to bear fields of the order of $10^{13}-10^{15} \mathrm{G}$ at their surface [19], reaching values several orders of magnitude greater at their center [20-22]. Moreover, such strong magnetic fields are also expected in present and future experiments at the RHIC, NICA and FAIR facilities, designed to probe the phase diagram of strongly interacting matter at low temperatures and intermediate to large densities.

So far, the effect of a magnetic field on the BEC/BCS crossover has been analyzed in the context of a two-channel model [23], where fermion and boson degrees of freedom are introduced from the beginning in the corresponding effective Lagrangian density. The aim of the present study is to go beyond this by considering a single-channel model. Since this is already a quite complicated issue by itself, we will work in the framework of the two-color NJL model, where the existence of a BEC-BCS crossover for conventional model parametrizations is firmly established. We recall here that, in contrast to what happens in $N_{c}=3$ QCD, this theory allows for lattice simulations at finite chemical potential. Results from this model regarding the diquark condensation in the absence of magnetic fields have been compared, e.g. in Ref. [12], with lattice simulations of $N_{c}=2$ QCD [16]. It turns out that the predictions of the NJL model for the diquark condensate are similar to those of chiral perturbation theory [24], as far as the agreement with lattice results are concerned. The NJL model is therefore interesting to investigate the general symmetry properties associated with two-color quark matter and their role in the phase transitions. Moreover, some aspects of the impact of the presence of external magnetic fields on the properties of two-color quark matter at finite temperature and vanishing chemical potential have been investigated using both effective models [25] and lattice simulations [17,18].

This paper is organized as follows. In Sec. II we introduce a two-color and two-flavor NJL-type model in the presence of a magnetic field and the corresponding thermodynamic potential to study the phase structure and, in particular, the BEC-BCS transition. Some possible regularization schemes used in the literature are also given. In Sec. III we give the results of our analysis and also make a comparison between different regularizations used when regarding the critical quantities and the crossover. In Sec. IV, we interpret the results obtained and check for their consistency. Our concluding remarks are given in Sec. V. One appendix is included, where some of the technical details are given.

\section{THE $N_{c}=2$ NJL MODEL IN THE PRESENCE OF AN EXTERNAL MAGNETIC FIELD}

We start by specifying the model and its parameters. We consider a two-color and two-flavor NJL-type model that includes scalar-pseudoscalar and color pairing interactions. The corresponding Lagrangian density, in the presence of an external electromagnetic field, is given by

$$
\begin{aligned}
\mathcal{L}= & \bar{\psi}\left(i D-m_{c}\right) \psi+G_{S}\left[(\bar{\psi} \psi)^{2}+\left(\bar{\psi} i \gamma_{5} \tau \psi\right)^{2}\right] \\
& +G_{D}\left(\bar{\psi} i \gamma_{5} \tau_{2} t_{2} C \bar{\psi}^{T}\right)\left(\psi^{T} C i \gamma_{5} \tau_{2} t_{2} \psi\right) .
\end{aligned}
$$

Here, $C=i \gamma_{0} \gamma_{2}$ is the charge conjugation matrix and $m_{c}$ is the current fermion mass, which we take to be equal for both flavors. Moreover, $\tau_{i}$ and $t_{i}$ are the Pauli matrices in flavor and color spaces, respectively. As usual, we will assume that the interaction terms are derived from an effective local coupling between color current terms. The two coupling constants $G_{S}$ and $G_{D}$, for the present twocolor NJL model [12] can be related simply as $G_{S}=$ $G_{D}=G$. This relation has to be imposed in order to have the symmetries of two-color QCD, and must be satisfied regardless of the type of the effective interaction that we start from. The coupling of the quarks to the electromagnetic field $\mathcal{A}_{\mu}$ is implemented through the covariant derivative, $D_{\mu}=\partial_{\mu}-i Q \mathcal{A}_{\mu}$, where $Q$ is the usual quark charge matrix $Q=\operatorname{diag}\left(q_{u}, q_{d}\right)$. In the present $N_{c}=2$ model we choose the charges to be $q_{u}=e$ and $q_{d}=-e$. In this way, as in Ref. [23], the charged fermions can be considered to mimic the rotated charged quarks that pair to form the electrically neutral Cooper pairs in actual QCD. Note, however, that while in the latter case quark matter will still behave as a color superconductor, two-color Cooper pairs are color singlets. Therefore, in the two-color 
QCD we are dealing with a true superfluid where just a global (baryon number) symmetry is spontaneously broken. Finally, we consider that the system is in the presence of a static and constant magnetic field in the third direction, namely, we take $\mathcal{A}_{\mu}=\delta_{\mu 2} x_{1} B$.

\section{A. The thermodynamical potential in the mean-field approximation}

At vanishing magnetic field, the mean-field thermodynamic potential at temperature $T$ and chemical potential $\mu$ is given by [7]

$\Omega=\Omega_{0}-8 T \sum_{s= \pm 1} \int \frac{d^{3} k}{(2 \pi)^{3}} \ln \left\{1+\exp \left[-\frac{\sqrt{\left(E_{k}+s \mu\right)^{2}+\Delta^{2}}}{T}\right]\right\}$

where $\Omega_{0}$ is the zero-temperature contribution,

$$
\begin{aligned}
\Omega_{0}= & \frac{\left(m-m_{c}\right)^{2}+\Delta^{2}}{4 G} \\
& -4 \sum_{s= \pm 1} \int \frac{d^{3} k}{(2 \pi)^{3}} \sqrt{\left(E_{k}+s \mu\right)^{2}+\Delta^{2}},
\end{aligned}
$$

and $E_{k}=\sqrt{k^{2}+m^{2}}$, with $m$ being the dressed quark mass.

The addition of a finite constant magnetic field can be easily implemented in the above expressions by considering the following substitutions [26]:

$$
\begin{aligned}
2 \int \frac{d^{3} k}{(2 \pi)^{3}} & \rightarrow \sum_{f=u}^{d} \frac{\left|q_{f}\right| B}{4 \pi} \sum_{l=0}^{\infty} \alpha_{l} \int_{-\infty}^{+\infty} \frac{d k_{3}}{2 \pi}, \\
E_{k} & \rightarrow E_{k_{3}, l}=\sqrt{k_{3}^{2}+2 l\left|q_{f}\right| B+m^{2}},
\end{aligned}
$$

where $\alpha_{l}=2-\delta_{l, 0}$ takes into account the degeneracy of the Landau levels. Therefore, the resulting zerotemperature mean-field thermodynamic potential in the presence of a constant magnetic field reads

$$
\Omega_{0}(m, \Delta, B, \mu)=\frac{\left(m-m_{c}\right)^{2}+\Delta^{2}}{4 G}-2 \sum_{f=u}^{d} \frac{\left|q_{f}\right| B}{4 \pi} \sum_{s= \pm 1} \sum_{l=0}^{\infty} \alpha_{l} \int_{-\infty}^{+\infty} \frac{d k_{3}}{2 \pi} \sqrt{\left(E_{k_{3}, l}+s \mu\right)^{2}+\Delta^{2}} .
$$

In the following, we will discuss the different ways to regularize the thermodynamic potential when including the effects of an external magnetic field.

\section{B. Model parameters and regularization schemes}

Since the model under consideration is nonrenormalizable, a proper regularization scheme is required to avoid ultraviolet divergences. As it will be discussed below for the regularization procedures to be used in this work, this implies the existence of a cutoff parameter $\Lambda$. Thus, the coupling constant $G=G_{S}=G_{D}$, the current quark mass $m_{c}$ and $\Lambda$ form a set of three parameters that must be specified in order to proceed with the numerical calculations. In the case of the $N_{c}=3 \mathrm{NJL}$ model analysis, these parameters are usually fixed such as to reproduce the empirical values in vacuum for the pion mass $m_{\pi}$, the pion decay constant $f_{\pi}$ and for the quark condensate $\langle\bar{q} q\rangle_{0}$. The situation for the $N_{c}=2$ case is not, however, so clear. Here, we follow the procedure proposed in Ref. [13], which is based on the $N_{c}$ scaling of physical quantities. Using the fact that $f_{\pi}$ is proportional to $\sqrt{N_{c}}$ and the chiral condensate to $N_{c}$, we rescale the three-color values by factors of $\sqrt{2 / 3}$ and $2 / 3$, respectively. Namely, we choose the model parameters such as to reproduce the values $f_{\pi}=$ $75.45 \mathrm{MeV}, m_{\pi}=140 \mathrm{MeV}$ and $\langle\bar{q} q\rangle_{0}^{1 / 3}=-218 \mathrm{MeV}$.

Let us now turn to the choice of the regularization scheme. One possibility is to introduce a form factor $U_{\Lambda}$ such that $\sum_{l=0}^{\infty} \int_{-\infty}^{\infty} \frac{d k_{3}}{2 \pi} \rightarrow \sum_{l=0}^{\infty} \int_{-\infty}^{\infty} \frac{d k_{3}}{2 \pi} U_{\Lambda}\left(\sqrt{k_{3}^{2}+2 l\left|q_{f}\right| B}\right)$.

In fact, most of the studies of the effect of magnetic fields within NJL-type models that include color pairing interactions are based on this kind of regularization (see, for example, Refs. [27-30]). As for the explicit form of the regularization function one might, in principle, be tempted to use a simple step function $\theta(x-\Lambda)$. It is known, however, that this procedure introduces strong unphysical oscillations in the behavior of different quantities as functions of the magnetic field. A discussion on this can be found in, e.g., Refs. [31-33], where it was also observed that the use of smooth regulator functions improves the situation. In fact, this allows one to identify possible physical oscillations appearing in some cases [27,28]. Different smooth form factors have been used in the literature. For example, in Ref. [32] Lorentzian functions of the form

$$
U_{\Lambda}^{(\operatorname{Lor} N)}(x)=\left[1+\left(\frac{x^{2}}{\Lambda^{2}}\right)^{N}\right]^{-1},
$$

have been used. Alternatively, in Ref. [29], Wood-Saxontype form factors like

$$
U_{\Lambda}^{(W S \alpha)}(x)=\left[1+\exp \left(\frac{x / \Lambda-1}{\alpha}\right)\right]^{-1},
$$


were introduced. Note that the form factor in the above expressions is chosen in such a way that three-momentum cutoff is used concomitant with the introduction of the magnetic field. In particular, note that the limit of $B \rightarrow 0$ reproduces the usual three-momentum cutoff in the simpler case of a step function regularization. But due to the aforementioned problem with this regularization, it has motivated these other forms of regularization in the literature. It should also be noted that all these form factors include a constant that regulates its smoothness. To choose the values for such a constant one is limited by the fact that a too steep function leads to the unphysical oscillations already mentioned, while a too smooth function leads to values of the quark condensate in the absence of the magnetic field that are quite above the phenomenological range. Thus, the value $N=5$ is usually chosen in the case of the Lorentzian form factor, while $\alpha=0.05$ is taken for the case of the Wood-Saxon one. In the following, we will identify these two regularization cases as Lor5 and WS0.05, respectively.

An alternative way to regularize the finite- $B$ thermodynamic potential was suggested in Ref. [34]. Within this procedure, the terms in the thermodynamic potential that are explicitly dependent on the magnetic field turn out to be finite and, thus, only those terms that are independent of it need to be regularized. In a way, this so-called "magneticfield-independent regularization" (MFIR) can be considered as an extension of the method described in, e.g., Ref. [26] to the case in which color pairing interactions are present.

Following the regularization procedure described in Ref. [34], after summing and subtracting convenient terms, the thermodynamic potential (2.5) can be cast into the form (see also the Appendix for details)

$$
\begin{aligned}
\Omega_{0}(m, \Delta, B, \mu)= & \Omega_{0}-\frac{N_{c}}{2 \pi^{2}} \sum_{f=u}^{d}\left(\left|q_{f}\right| B\right)^{2}\left\{\zeta^{\prime}\left(-1, x_{f}\right)-\frac{1}{2}\left(x_{f}^{2}-x_{f}\right) \ln \left(x_{f}\right)+\frac{x_{f}^{2}}{4}\right\} \\
& -\frac{N_{c}}{4 \pi^{2}} \sum_{f=u}^{d}\left(\left|q_{f}\right| B\right) \int_{0}^{\infty} d k_{3}\left\{\sum_{l=0}^{\infty} \alpha_{l} F\left(k_{3}^{2}+2 l\left|q_{f}\right| B\right)-2 \int_{0}^{\infty} d y F\left(k_{3}^{2}+2 y\left|q_{f}\right| B\right)\right\},
\end{aligned}
$$

where

$F\left(z^{2}\right)=\sum_{s= \pm 1}\left[\sqrt{\left(\sqrt{z^{2}+m^{2}}+s \mu\right)^{2}+\Delta^{2}}-\sqrt{z^{2}+m^{2}+\Delta^{2}}\right]$,

and $x_{f}=\left(m^{2}+\Delta^{2}\right) /\left(2\left|q_{f}\right| B\right)$. In this expression, a threedimensional sharp cutoff $\Lambda$ can be introduced to regularize $\Omega_{0}$, which has no explicit magnetic field dependence, while the other terms are ultraviolet finite. The details concerning the derivation of Eq. (2.9) can be found in Ref. [34]. For completeness, the main ones are also given in the Appendix.

For each of the regularization schemes mentioned above, Lor5, WS0.05, as well as for the MFIR case, we need to evaluate the parameters that reproduce the scaled physical values of $f_{\pi}, m_{\pi}$ and $\langle\bar{q} q\rangle_{0}$, in the $N_{c}=2$ case. The procedure to obtain these quantities is discussed in detail in Ref. [5]. The values obtained and used in this work are shown in Table I.

TABLE I. Parameter sets we have used for the two-color NJL $\mathrm{SU}_{f}(2)$ model. Here, $m(0)$ stands for the dressed quark mass at $\mu=B=0$.

\begin{tabular}{lcccc}
\hline \hline Parameter set & $m(0)(\mathrm{MeV})$ & $m_{c}(\mathrm{MeV})$ & $G\left(\mathrm{GeV}^{-2}\right)$ & $\Lambda(\mathrm{MeV})$ \\
\hline Lor5 & 337.232 & 5.398 & 8.00 & 616 \\
WS0.05 & 311.865 & 5.401 & 7.39 & 650 \\
MFIR & 305.385 & 5.400 & 7.23 & 657 \\
\hline \hline
\end{tabular}

\section{NUMERICAL RESULTS}

In the previous section we gave the mean-field thermodynamic potential in the presence of an external magnetic field, Eq. (2.5), and presented some different regularization schemes to avoid the divergences. Given the corresponding regularized form $\Omega_{0}^{\text {reg }}(m, \Delta, B, \mu)$, the associated gap equations for $m$ and $\Delta$ then read

$$
\frac{\partial \Omega_{0}^{\mathrm{reg}}(m, \Delta, B, \mu)}{\partial m}=0, \quad \frac{\partial \Omega_{0}^{\mathrm{reg}}(m, \Delta, B, \mu)}{\partial \Delta}=0 .
$$

In what follows we present and discuss the results obtained by solving numerically these equations. ${ }^{1}$

\section{A. Behavior of the order parameters}

It is known that at zero chemical potential the magnetic catalysis effect is present (see, for example, Ref. [35]). This is seen in Fig. 1(a), where the behavior of $m$ as a function of the magnetic field is shown, for all three regularization schemes studied in this work. This coincides with the result obtained in the NJL model without the diquark interaction since $\Delta$ vanishes in this case. For $B=0$, a transition to a $\Delta \neq 0$ phase occurs at $\mu=m_{\pi} / 2[4,7]$, or, equivalently, at

\footnotetext{
${ }^{1}$ In our notation $m_{\pi}=0.14 \mathrm{GeV}$ and $m_{\pi}(e B)$ is a function of the magnetic field.
} 

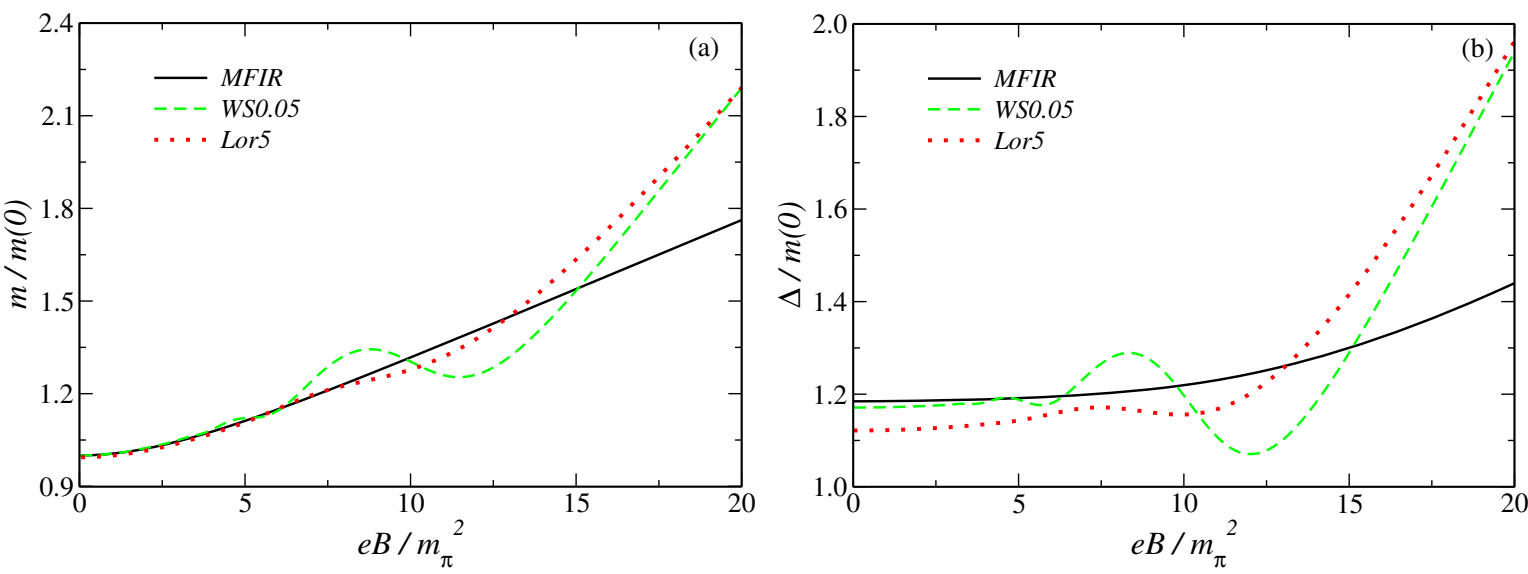

FIG. 1. Results for (a) the effective quark mass $m$, at $\mu=0$, and (b) the diquark condensate $\Delta$, at $\mu_{B}=6 m_{\pi}$, as functions of the magnetic field for the three regularization schemes (see Table I). Both $m$ and $\Delta$ are scaled by $m(0) \equiv m(\mu=e B=0)$.

$\mu_{B}=m_{\pi}$. Here, $\mu_{B}=2 \mu$ is the baryon chemical potential for the $N_{c}=2$ case we are dealing with. Thus, by fixing the chemical potential at a value $\mu_{B}>m_{\pi}$ a nonvanishing value for $\Delta$ is obtained. The corresponding behavior as a function of the magnetic field is shown in Fig. 1(b) for the case for a value of $\mu_{B}=6.0 m_{\pi}(\equiv 0.84 \mathrm{GeV}$ ) (which is chosen as a representative value for illustrative purposes) and the three regularizations schemes, WS0.05, Lor5 and MFIR.

The aforementioned oscillations are clearly seen in both panels in Fig. 1, i.e., in both the effective quark mass and the diquark condensate, when using the WS0.05 and Lor5 regularization schemes. We note that they are somewhat smaller in the second case, which corresponds to a smoother regulator. These nonphysical oscillations can be traced to the fact that the regularization procedure depends explicitly on the magnetic field. Therefore, they disappear when the divergent terms are disentangled from the magnetic contributions by using the MFIR scheme. It is also clear from both panels in Fig. 1 that the WS0.05 and Lor5 regularization schemes start to deviate strongly from the MFIR scheme at large values of the magnetic field, $e B / m_{\pi}^{2} \gtrsim 15$. With respect to this, it should be borne in mind that some estimates [22] indicate that the magnetic fields at the center of magnetars can be as large as $e B / m_{\pi}^{2} \simeq 30$.

We consider now the behaviors of $\Delta$ and the effective quark mass as functions of the baryon chemical potential for different values of the magnetic field. This is shown in Fig. 2. We restrict ourselves to the results for the MFIR and WS0.05 regularizations, since the results for Lor5 are qualitatively similar to those for the WS0.05. There is a chirally broken phase with $\Delta=0$ for low enough chemical potentials and a BEC phase in which $\Delta$ is finite and chiral symmetry is partially restored. The transition connecting these two phases is of second-lorder and, when $B=0$, it occurs at $\mu_{B}=m_{\pi}$. This result is in agreement with those obtained using chiral effective theories [16]. This result also remains true for both schemes presented here. The magnetic catalysis effect can also be observed, where both $m$ and $\Delta$ are seen to increase with the magnetic field. We also note
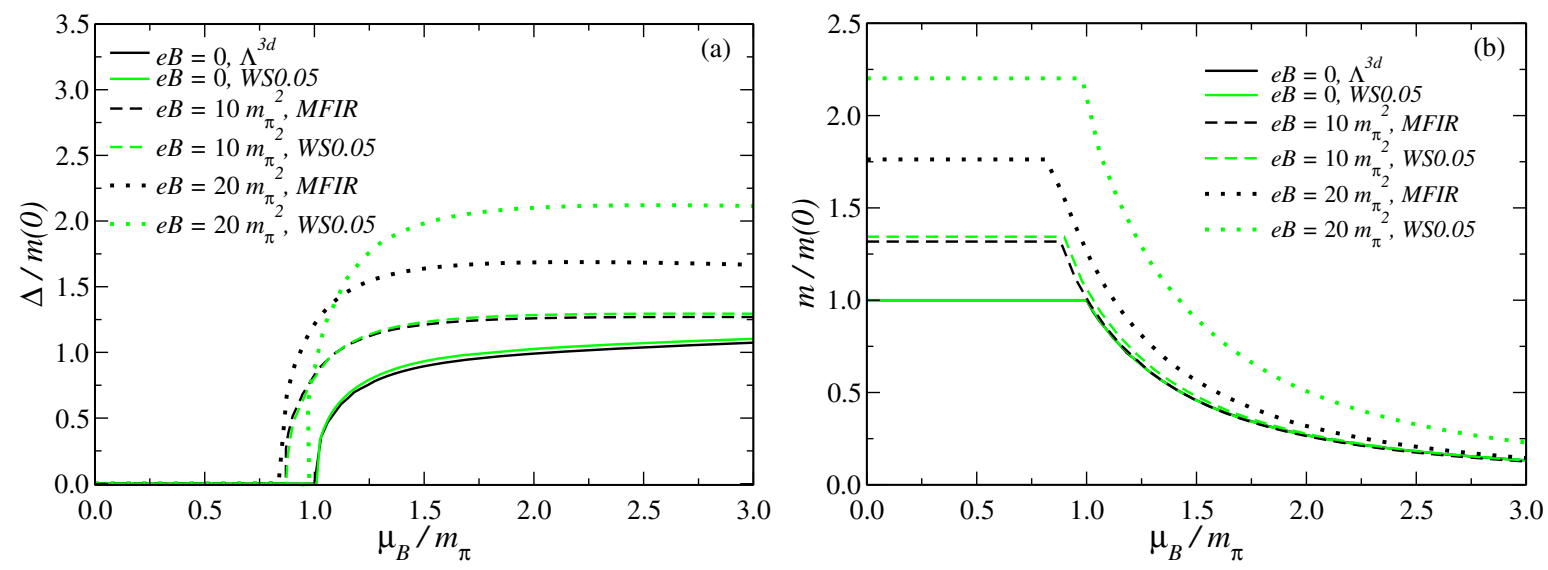

FIG. 2. Results for (a) the diquark condensate $\Delta$, and (b) the effective quark mass $m$, both as a function of the baryon chemical potential, $\mu_{B}$ (where $\mu_{B}=N_{c} \mu$ with $N_{c}=2$ ), in the WS0.05 and MFIR schemes. 
that for $B=0$ (corresponding to the lowest curves in both panels of Fig. 2), the MFIR regularization exactly reduces to the traditional three-dimensional cutoff $\Lambda$, indicated by the $\Lambda^{3 d}$ label in the figures, and that the results obtained for both regularization methods are similar for $e B=0$ and $10 m_{\pi}^{2}$, but differ significantly for $e B=20 m_{\pi}^{2}$.

\section{B. The critical chemical potentials}

We now determine how the magnetic field affects the critical baryon chemical potential $\mu_{B_{c}}^{\mathrm{BEC}}$ for the diquark condensate (BEC) phase transition and for the BEC-BCS

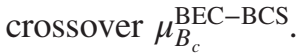

Since the phase transition for diquark condensation remains second order even in the presence of an external magnetic field, we can obtain the corresponding critical baryon chemical potential by using a Ginzburg-Landau expansion for the thermodynamic potential (2.5) around the critical point [14],

$$
\begin{aligned}
\Omega_{0}\left(m, \Delta, B, \mu_{B} / 2\right)= & \Omega_{0}\left(m, 0, B, \mu_{B} / 2\right) \\
& +\alpha_{2}\left(m, B, \mu_{B} / 2\right)|\Delta|^{2} \\
& +\alpha_{4}\left(m, B, \mu_{B} / 2\right)|\Delta|^{4}+\mathcal{O}\left(|\Delta|^{6}\right),
\end{aligned}
$$

where the Ginzburg-Landau coefficients $\alpha_{n}$ are defined as

$$
\alpha_{n}(m, B, \mu)=\left.\frac{1}{n !} \frac{d^{n} \Omega_{0}\left(m, \Delta, B, \mu_{B} / 2\right)}{d \Delta^{n}}\right|_{\Delta=0} .
$$

As customary, the critical chemical potential for the BEC transition can be obtained from the condition $\alpha_{2}(m, B$, $\left.\mu_{B_{c}}^{\mathrm{BEC}} / 2\right)=0$, where $m$ results from minimizing the first term in Eq. (3.2) for each value of $e B$.

We now turn to the determination of the critical chemical potential for the BEC-BCS crossover. For this purpose, it is convenient to define a reference chemical potential, $\mu_{N}=\mu_{B} / 2-m$, in terms of which the transition between the two states is characterized by the condition $\mu_{N}=0$ [7]. The rationale behind this is as follows. Considering for simplicity the case $B=0$, a typical dispersion relation is given by

$$
E_{\Delta}^{ \pm}=\sqrt{\left(\sqrt{\vec{k}^{2}+m^{2}} \pm \frac{\mu_{B}}{2}\right)^{2}+\Delta^{2}},
$$

where $E_{\Delta}^{-}$corresponds to particle and $E_{\Delta}^{+}$corresponds to antiparticle excitations. For small $\mu_{B}$ we have $\mu_{N}<0$, and the minimum of the dispersion is located at $|\vec{k}|=0$, with particle gap energy $\sqrt{\mu_{N}^{2}+\Delta^{2}}$. This corresponds to the fermionic (quark) spectrum in the BEC state. On the other hand, at larger values of $\mu_{B}$, we have $\mu_{N}>0$. The minimum of the dispersion is shifted to $|\vec{k}|=\mu_{B} / 2$ and the particle gap is $\Delta$. This corresponds to the fermionic spectrum in the

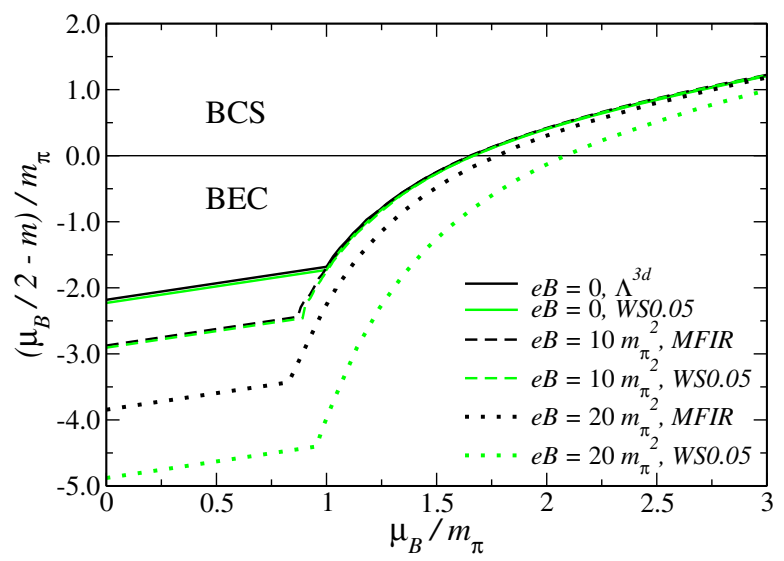

FIG. 3. The reference chemical potential, $\mu_{N}=\mu_{B} / 2-m$, as a function of the chemical potential in the WS0.05 and MFIR regularization schemes.

BCS state. Thus, the condition $\mu_{N}=0$ can be used to determine the position of the crossover. Note that it implies $m=\mu_{B_{c}}^{\mathrm{BEC}-\mathrm{BCS}} / 2$. Therefore, the critical chemical potential can be obtained by setting this relation in the gap equations (3.1) and by solving them for $\Delta$ and $\mu_{B_{c}}^{\mathrm{BEC}-\mathrm{BCS}}$ for different values of $B$.

In Fig. 3 we show the reference chemical potential $\mu_{N}$ as a function of the chemical potential, for $e B=0,10 m_{\pi}^{2}$ and $20 m_{\pi}^{2}$. We have restricted ourselves again, for simplicity, to show results only for the MFIR and WS0.05 regularization schemes. Negative values of $\mu_{N}$ correspond to the BEC region, while positive values correspond to the BCS region. These two phases are indicated by the regions below and above, respectively, the thin horizontal solid line shown in Fig. 3. For the values of magnetic fields shown in Fig. 3, we find that the point for which $\mu_{N}$ changes sign, the BECBCS crossover, is shifted to the right, i.e., when increasing the value of the magnetic field, the crossover takes place at a larger value of $\mu$ (or, analogously, at a larger value of $\mu_{B}$ ). This corresponds to a strengthening of the BEC region. This phenomenon is observed to occur in both regularization schemes considered in this work.

Note that the magnetic field values considered in the figures, $e B=10 m_{\pi}^{2}$ and $20 m_{\pi}^{2}$ (corresponding to approximately 0.2 and $0.4 \mathrm{GeV}^{2}$ respectively) are usually referred to in the literature as strong magnetic fields. These values are representative of the fields expected in heavy-ion collision experiments, like in the RHIC and in the LHC, which are estimated to have magnitudes of the order of $e B \sim 15 m_{\pi}^{2}$ [36].

The numerical results for the baryon critical chemical potential for the BEC transition $\mu_{B_{c}}^{\mathrm{BEC}}$ are shown in Fig. 4(a). In the MFIR scheme, we see that from small to moderately strong values of $e B$ an inverse magnetic catalysis (IMC) effect is observed, i.e., the value of $\mu_{B_{c}}^{\mathrm{BEC}}$ decreases with the magnetic field, while for very strong magnetic fields, $\mu_{B_{c}}^{\mathrm{BEC}}$ grows rapidly with $e B$ in the MFIR scheme. The IMC 

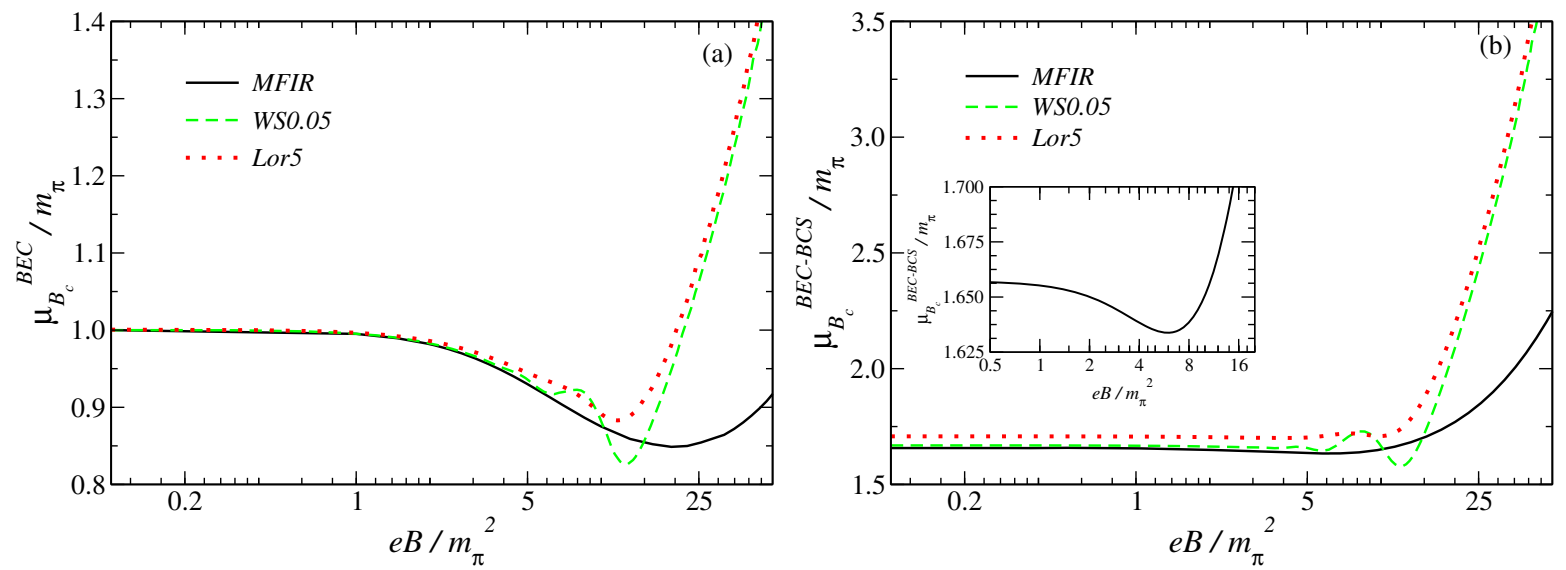

FIG. 4. Critical baryon chemical potential $\mu_{B}$ as a function of the magnetic field for (a) the diquark BEC phase transition and (b) the BEC-BCS crossover, in the WS0.05, Lor5 and MFIR regularization schemes. The inset in panel (b) shows the region for IMC for the MFIR case.

phenomenon was first observed in the NJL model in Ref. [37] at $T=0$ and in Ref. [38] for the full $T-\mu-$ $B$ case. Since then, it has been found and described in different models (see, e.g., Refs. [39,40] and references therein). For the parameters we have considered, the IMC effect is clearly seen in the interval $1 \lesssim e B / m_{\pi}^{2} \lesssim 20$.

The behavior of $\mu_{B_{c}}^{\mathrm{BEC}-\mathrm{BCS}}$ for the BEC-BCS crossover is shown in Fig. 4(b). Note that from small to moderate magnetic field values, $e B / m_{\pi}^{2} \lesssim 9$, the value of $\mu_{B_{c}}^{\mathrm{BEC}-\mathrm{BCS}}$ changes little. This indicates that the magnetic field does not tend to affect strongly the size of the BEC region in that range of values for the magnetic field. Yet, we can still see an IMC effect happening also for the crossover, which is shown in the inset in Fig. 4(b). However, this phenomenon happens over a much smaller range of magnetic fields and the variation of the critical baryon chemical potential is much smaller than the one in the BEC transition. On the other hand, for larger values of the magnetic fields, the $\mathrm{BEC}$ region increases with the magnetic field, as indicated by the rise of the critical chemical potential in Fig. 4(b). The reduced IMC effect in the BEC-BCS crossover can be qualitatively understood as a competition between magnetic catalysis, which always tends to increase the chiral mass $m$ (see Fig. 1), thus pushing the critical chemical potential up, and the IMC, which tends to facilitate the BEC transition. The overall result is an almost constant critical chemical potential from small to moderate values of the magnetic field and an increasing $\mu_{B_{c}}^{\mathrm{BEC}-\mathrm{BCS}}$ afterwards.

Finally, note also the differences in behaviors for the different regularization schemes seen in Fig. 4. Both WS0.05 and Lor5 regularization schemes produce again nonphysical oscillations similar to what we have seen before in Fig. 1. It is important to remark that, for the chosen parametrizations, there are no oscillations related to the van Alphen-de Haas (vA-dH) effect in this model. These oscillations do have a physical meaning and would usually be expected to appear as a consequence of the oscillations of the density of states near the Fermi surface. In the present case they are not visible because $\Delta$ takes rather large values in the crossover side, while in the BEC side the effective quark mass remains also rather large. As a consequence, the oscillations are washed away in both cases. Studies have been reported in which the oscillations are actually visible, owing to a smaller diquark coupling $[27,28]$ or because there are quark species that do not participate in the pairing [as would occur in the 2SC phase for color $\mathrm{SU}(3)$ ], which have ordinary vA-dH oscillations, which in turn produce oscillations indirectly on $\Delta$ [34]. In the later case, the quarks that are decoupled from the diquark gap produce vA-dH transitions, which are related to the change in population of Landau levels, and are nearly vertical in the phase diagram for chemical potential and magnetic field. Note also that the WS0.05 and Lor5 regularization schemes always overestimate the values for the critical baryon chemical potentials, both for the BEC second-order diquark condensate phase transition and also for the BEC-BCS crossover, with the difference increasing with the magnetic field. The reason for this is the same as already explained before with respect to the same behavior seen in Fig. 1.

\section{INTERPRETING THE RESULTS}

Let us better interpret the numerical results shown in the previous section. For the numerical results presented in this section, we restrict ourselves to showing only the MFIR scheme for simplicity.

First, let us show that the condition used to determine the BEC transition point remains valid. The transition point was determined by setting the coefficient for the quadratic term in the diquark field in Eq. (3.2) to zero, i.e., by making $\alpha_{2}\left(m, B, \mu_{B_{c}}^{\mathrm{BEC}} / 2\right)=0$. This condition is, of course, only valid if the transition is second order. We can verify that the transition is in fact second order and remains so in a finite 

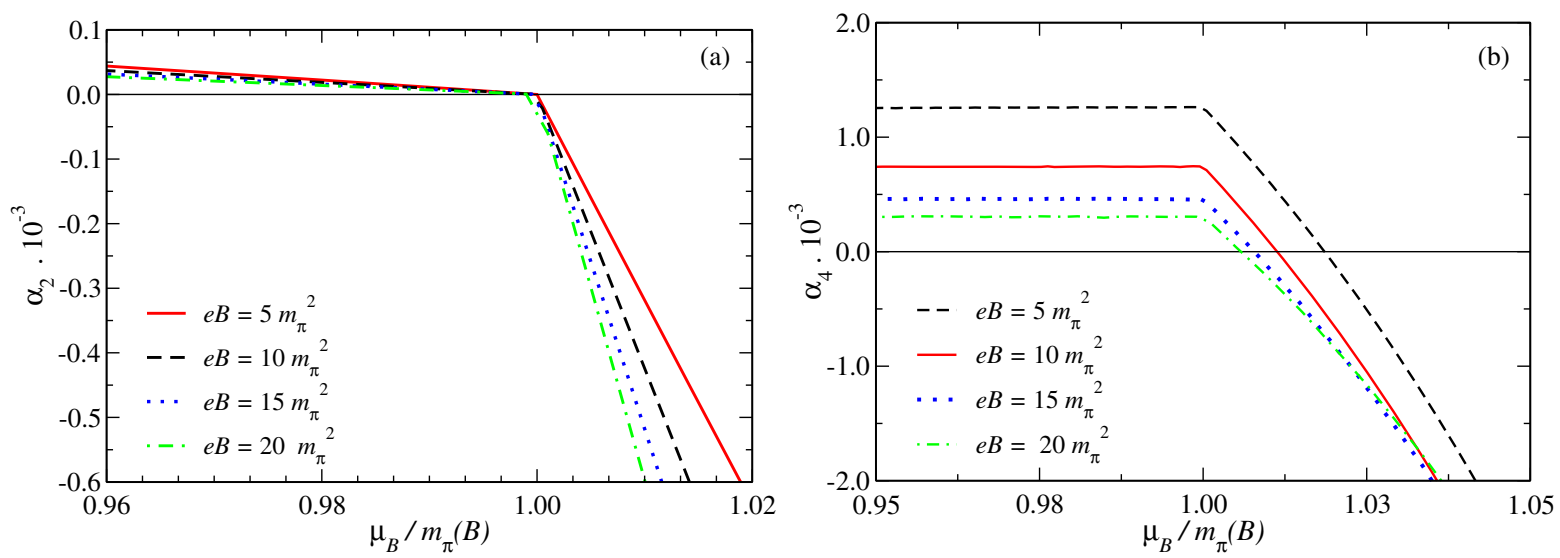

FIG. 5. The quadratic and quartic coefficients in the Ginzburg-Landau expansion for the thermodynamic potential, Eq. (3.2).

magnetic field by analyzing the coefficient for the quartic term in the diquark field in Eq. (3.2). If the quartic term is positive for the values of magnetic field considered, i.e., $\alpha_{4}\left(m, B, \mu_{B_{c}}^{\mathrm{BEC}} / 2\right)>0$ across the $\mathrm{BEC}$ transition, then the transition remains second order.

In Fig. 5 we plot both $\alpha_{2}\left(m, B, \mu_{B} / 2\right)$ and $\alpha_{4}(m, B$, $\left.\mu_{B} / 2\right)$ for a reasonable range of chemical potentials and for different values for the magnetic field. Note that on the horizontal axis in Fig. 5 we are using the baryon chemical potential normalized by the pion mass computed at a given value of magnetic field, $\mu_{B} / m_{\pi}(B)$. The reason for presenting the results this way will be fully justified below, where we explicitly give the expression defining the pion mass in a finite magnetic field, $m_{\pi}(B)$. As is well known, at $B=0$ the BEC transition for diquark condensation happens at $\mu_{B}=m_{\pi}$. As shown in the previous section, in the presence of a magnetic field the BEC transition tends to happen for a different value for $\mu_{B}$. The value for $\mu_{B}$ where the transition happens can be either smaller, in the case of IMC, or higher, as in the case where magnetic catalysis (MC) becomes dominant, than in the case where $B=0$. However, the BEC transition can be shown to always happen at the value of the pion mass where it is now determined at the given value of the magnetic field, $\mu_{B}=m_{\pi}(B)$. The plot in Fig. 5(a) indeed confirms this. It shows that $\alpha_{2}\left(m, B, \mu_{B} / 2\right)=0$ at $\mu_{B}=m_{\pi}(B)$ for all values of magnetic field.

Finally, in Fig. 5(b), we also show that $\alpha_{4}\left(m, B, \mu_{B} / 2\right)$ remains positive at the point where $\mu_{B}=m_{\pi}(B)$ and, therefore, the BEC transition remains second order at finite magnetic fields. We also notice from the results in Fig. 5 that $\alpha_{4}$ becomes negative for values of $\mu_{B}$ slightly higher than the BEC transition point. But this does not indicate that a first-order phase transition would follow the BEC transition, since this happens where $\alpha_{2}$ is already negative. Hence, no additional (local) vacuum values for the diquark field can be generated.

The dependence of the critical chemical potential for the BEC phase can be related to the magnetic field dependence of the diquark mass $m_{d}$ (at zero chemical potential). As the diquark is an electrically neutral particle, its mass should be insensitive to $B$ to leading order, and it will mildly decrease as $B$ gets bigger. In the $N_{c}=2$ case we are dealing with, at $\mu=0$ the diquark is degenerated with $\pi_{0}$ and we can analyze what happens with $m_{d}$ by looking at the neutral pion mass [41]. As a side observation concerning this degeneracy between the scalar diquarks with the neutral pion, we can interpret it based on some symmetry arguments satisfied by the model. Note that in the absence of the magnetic field the symmetry group of the present NJL model is $S p(4)$ (see, e.g., Ref. [24] for associated analysis in two-color QCD) with scalar diquarks and pions lying in the corresponding five-dimensional irrep. This symmetry, however, gets broken in the presence of the magnetic field, since the charged particles acquire a different mass than the neutral ones. Thus, the five-dimensional irrep goes to a three-dimensional one for the neutral sector, containing the two diquarks and the neutral pion.

The neutral pion mass decreases in real QCD, and it justifies our IMC results shown in Fig. 4(a) (at least below $10 m_{\pi}^{2}$ ). A similar situation is seen to happen in real QCD with isospin chemical potentials (see, e.g., Ref. [42]), where the BEC phase also contains charged pions. Accordingly, the magnetic field enhances the critical chemical potential, since the charged pion mass increases with $B$.

To further understand the IMC seen in the numerical results shown in Fig. 4, we can first establish a relation between the critical baryon chemical potential and the effective mass for the (neutral) pion field. Note that in the absence of the magnetic field the BEC transition happens at $\mu_{B_{c}}^{\mathrm{BEC}}=m_{\pi}$, as is well known and explicitly seen from the results of the previous section. As we have also shown above, at a finite magnetic field this transition also remains of second order. It is natural to then expect that the condition for the transition, $\mu_{B_{c}}^{\mathrm{BEC}}=m_{\pi}$, remains valid also at finite magnetic field values if we replace the vacuum pion mass by its effective value at some magnetic field, i.e., $m_{\pi}(B)$. Since the BEC transition is for diquark condensation and the 

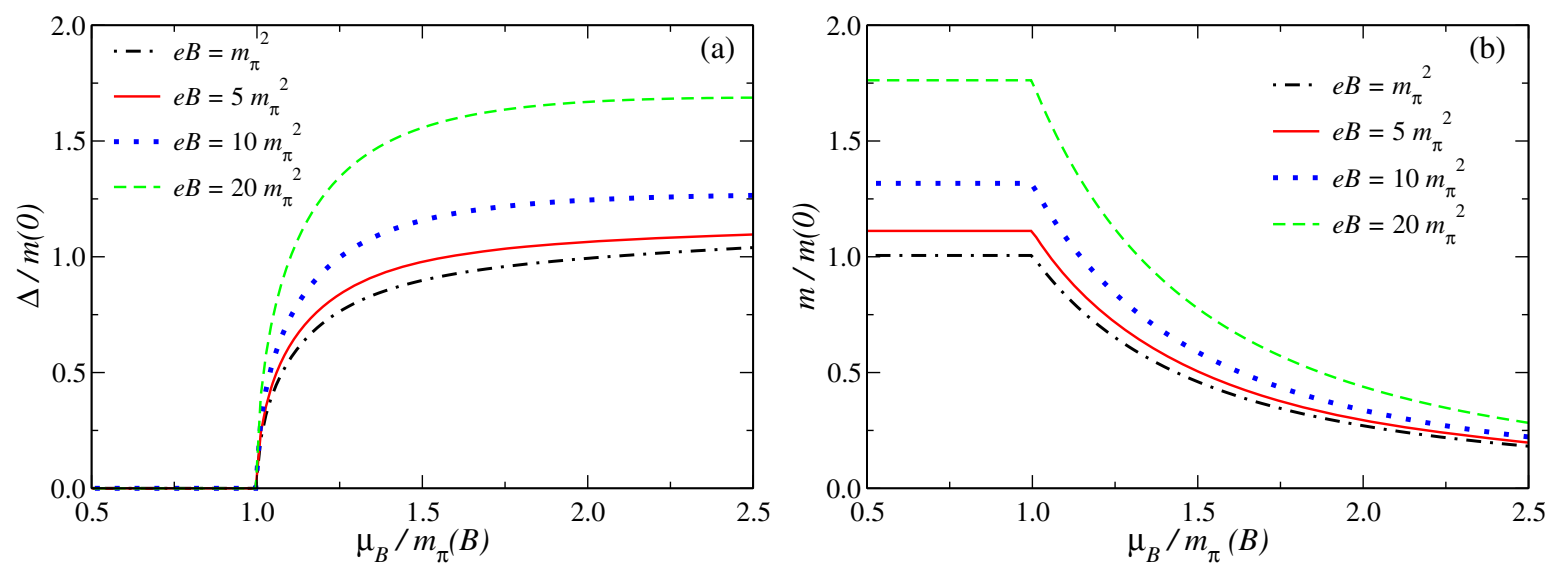

FIG. 6. (a) Diquark condensate $\Delta$ and (b) effective quark mass $m$, both as a function of the baryon chemical potential $\mu_{B}$ in the MFIR scheme. Here the pion mass used to rescale $\mu_{B}$ is a function of the magnetic field $m_{\pi}(B)$.

diquarks are neutral here, we can try to relate the transition point directly in terms of the neutral pion effective mass.

The effective pion mass is determined from its inverse propagator, which in momentum space-time can be expressed as

$$
G_{\pi}\left(p_{0}, \vec{p}\right)=-\frac{1}{2 G}-\Pi_{\pi}\left(p_{0}, \vec{p}\right)
$$

where $\Pi_{\pi}\left(p_{0}, \vec{p}\right)$ is the self-energy contribution for the pion, coming from a quark-antiquark loop at leading oneloop order. Since we are interested in the pion mass, we evaluate the self-energy in the rest frame, $\vec{p}=0$ and then set the energy at the mass shell, $p_{0}=m_{\pi}$, where $m_{\pi}$ is to be interpreted as the effective pion mass. The effective pion mass is then determined by the pion pole condition, $G_{\pi}\left(p_{0}=m_{\pi}, \vec{p}=0\right)=0$, or

$$
\frac{1}{2 G}=-\Pi_{\pi}\left(p_{0}=m_{\pi}, \vec{p}=0\right) .
$$

In the rest frame and computed at zero temperature, the one-loop self-energy for the pion in the present two-color NJL model can be expressed as [43]

$$
\begin{aligned}
& \Pi_{\pi}\left(p_{0}=m_{\pi}, \vec{p}=0\right) \\
& \quad=4 N_{c} \int \frac{d^{3} k}{(2 \pi)^{3}} \frac{E_{\Delta}^{+} E_{\Delta}^{-}+\epsilon_{k}^{+} \epsilon_{k}^{-}+\Delta^{2}}{m_{\pi}^{2}-\left(E_{\Delta}^{+}+E_{\Delta}^{-}\right)^{2}}\left(\frac{1}{E_{\Delta}^{+}}+\frac{1}{E_{\Delta}^{-}}\right),
\end{aligned}
$$

where we have defined $E_{\Delta}^{ \pm}=\sqrt{\left(\epsilon_{k}^{ \pm}\right)^{2}+\Delta^{2}}, \epsilon_{k}^{ \pm}=E_{k} \pm \mu$ and $E_{k}=\sqrt{k^{2}+m^{2}}$, as also defined earlier in Sec. II. At the BEC transition point for diquark condensation, we can set $\Delta=0$ in Eq. (4.3) and the pion pole condition becomes

$$
\frac{1}{2 G}=16 N_{c} \int \frac{d^{3} k}{(2 \pi)^{3}} \frac{E_{k}}{4 E_{k}^{2}-m_{\pi}^{2}} .
$$

To introduce the magnetic field, we can follow the prescription as given in Eq. (2.4), to then obtain that

$$
\frac{1}{2 G}=8 N_{c} \sum_{f=u}^{d} \frac{\left|q_{f}\right| B}{4 \pi} \sum_{l=0}^{\infty} \alpha_{l} \int_{-\infty}^{+\infty} \frac{d k_{3}}{2 \pi} \frac{E_{k_{3}, l}}{4 E_{k_{3}, l}^{2}-m_{\pi}^{2}} .
$$

An explicit derivation of the pion one-loop polarization term at zero temperature and in an external magnetic field can be found in Ref. [41]. In that work the authors evaluated the masses of $\sigma$ and $\pi_{0}$, the $\pi^{0}$ decay constant in the presence of a magnetic field at vanishing temperatures and baryon densities. In particular, they showed that the Gell-MannOakes-Renner relation remains valid in a magnetic medium.

We can now compare the pion pole condition (4.5) with the one determining the BEC transition point, which is determined by setting the coefficient of the quadratic term in the diquark field in Eq. (3.2) to zero, $\alpha_{2}\left(m, B, \mu_{B_{c}}^{\mathrm{BEC}} / 2\right)=0$. Using Eq. (3.3), we obtain that

$$
\begin{aligned}
& \alpha_{2}\left(m, B, \mu_{B_{c}}^{\mathrm{BEC}} / 2\right)=0 \\
& =\frac{1}{4 G}-\frac{N_{c}}{2} \sum_{f=u}^{d} \frac{\left|q_{f}\right| B}{4 \pi} \sum_{l=0}^{\infty} \alpha_{l} \int_{-\infty}^{+\infty} \frac{d k_{3}}{2 \pi} \\
& \quad \times\left[\frac{1}{E_{k_{3}, l}+\mu_{B_{c}}^{\mathrm{BEC}} / 2}+\frac{1}{E_{k_{3}, l}-\mu_{B_{c}}^{\mathrm{BEC}} / 2}\right] .
\end{aligned}
$$

The above equation can be rewritten in the form

TABLE II. The mass $m$ and diquark $\Delta$ in the two phases of the theory.

\begin{tabular}{lcc}
\hline \hline phase & $m$ & $\Delta$ \\
\hline$\mu_{B}<m_{\pi}(B)$ & $m(B, 0)$ & 0 \\
$\mu_{B} \geq m_{\pi}(B)$ & $m(B, 0)\left[\frac{m_{\pi}(B)}{\mu_{B}}\right]^{2}$ & $m(B, 0) \sqrt{1-\left[\frac{m_{\pi}(B)}{\mu_{B}}\right]^{4}}$ \\
\hline \hline
\end{tabular}



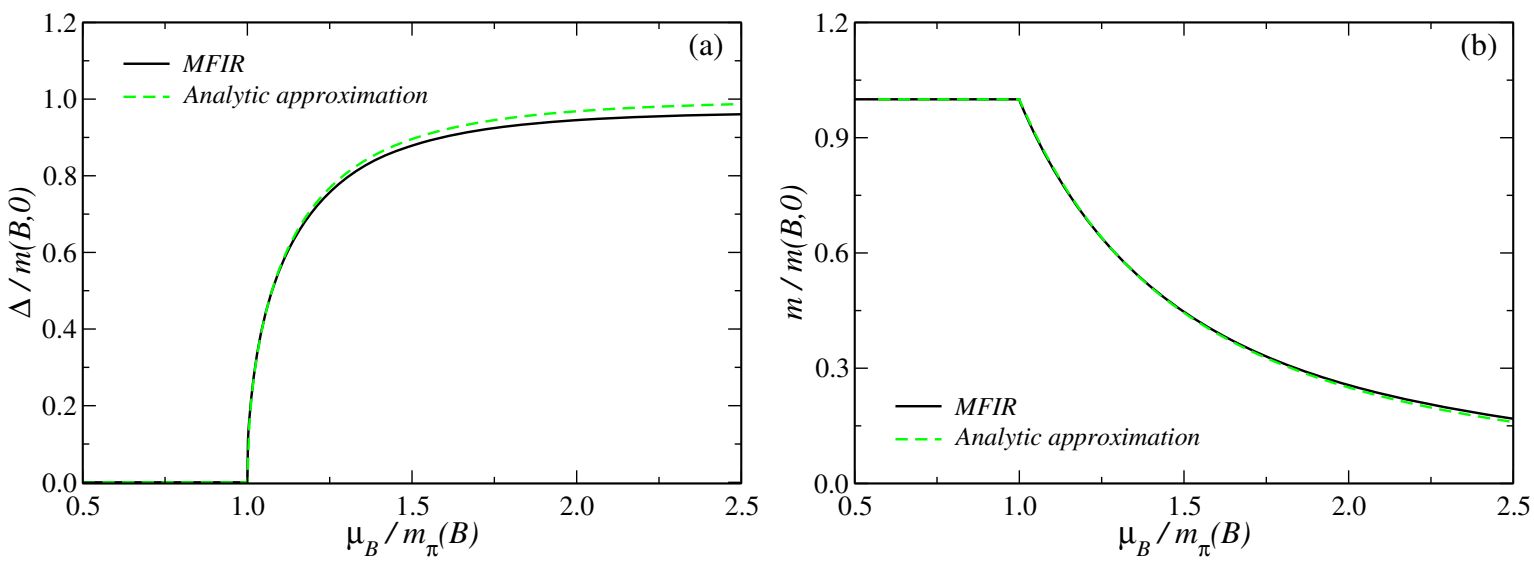

FIG. 7. (a) Diquark condensate $\Delta$ and (b) effective quark mass $m$, both as a function of the baryon chemical potential $\mu_{B}$ for $e B=10 m_{\pi}^{2}$. Solid lines are evaluated with the MFIR scheme and dashed lines are for our analytical approximation.

$$
\frac{1}{2 G}=8 N_{c} \sum_{f=u}^{d} \frac{\left|q_{f}\right| B}{4 \pi} \sum_{l=0}^{\infty} \alpha_{l} \int_{-\infty}^{+\infty} \frac{d k_{3}}{2 \pi} \frac{E_{k_{3}, l}}{4 E_{k_{3}, l}^{2}-\left(\mu_{B_{c}}^{\mathrm{BEC}}\right)^{2}} .
$$

Comparing Eq. (4.5) with Eq. (4.7) it becomes clear that the $\mathrm{BEC}$ condition for diquark condensation, $\mu_{B_{c}}^{\mathrm{BEC}}=m_{\pi}$, also remains valid at finite magnetic field, i.e., $\mu_{B_{c}}^{\mathrm{BEC}}=m_{\pi}(B)$, where $m_{\pi}(B)$ is the solution of Eq. (4.5) along the BEC transition.

In Fig. 6 we show our numerical results for the diquark condensate $\Delta$ and for the effective quark mass $m$ as a function of $\mu_{B}$ for different values of the magnetic field. Note that we have normalized $\mu_{B}$ with respect to $m_{\pi}(B)$. We can then observe that indeed the BEC transition always happens at $\mu_{B_{c}}^{\mathrm{BEC}}=m_{\pi}(B)$.

In Refs. $[15,44]$ the authors showed analytically that the "chiral rotation" behavior predicted by chiral perturbation

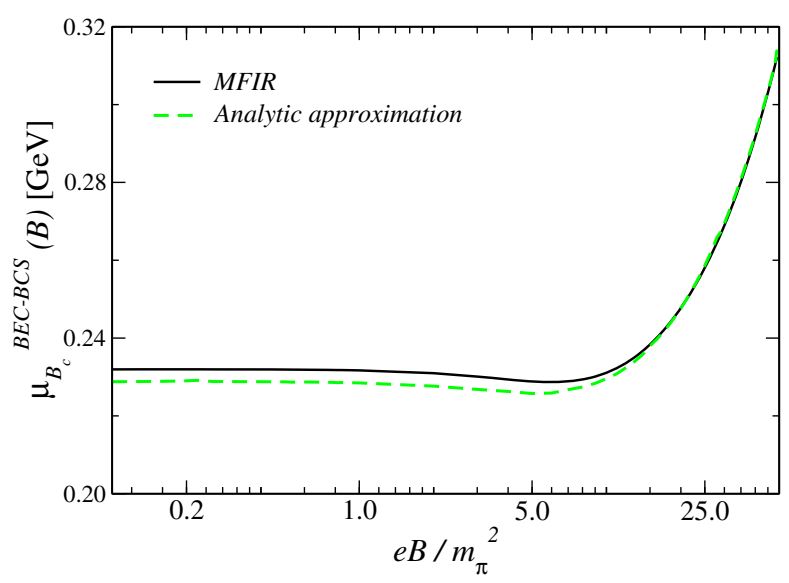

FIG. 8. The analytical approximation, Eq. (4.8) (dashed line) and the numerical result (solid line) for the magnetic field dependence of the critical chemical potential for the crossover $\mathrm{BEC}-\mathrm{BCS} \mu_{B}^{\mathrm{BEC}-\mathrm{BCS}}(B)$ (here expressed in units of $\mathrm{GeV}$ ). theories (ChPTs) [12,13,45] is valid in the NJL model near the quantum phase transition. The ChPT results for the diquark condensate and effective quark mass at $B=0$ can be found for example in Table 3 of Ref. [24]. We have verified that the relations found in the framework of ChPT [24] at vanishing magnetic field are also very well satisfied in our case at finite values of $B$. The relevant expressions at finite $B$ are given in Table II, where $m(B, 0)=$ $m\left(B, \mu_{B}=0\right)$.

In Fig. 7 we numerically verify the validity of the relations given in Table II at finite values of the magnetic field. The difference between the approximate relations and the numerical results are at the percentage level.

Having better understood the results for the BEC transition, we can now move to the case of the BEC-BCS crossover. The authors of Ref. [7] gave a very simple approximate analytic expression for the position of the BEC-BCS crossover [see, for instance, Eq. (40) in that reference], which only depends on the constituent quark mass and on the pion mass in the vacuum, $\mu_{B}^{0}=$ $\left[2 m(0) m_{\pi}^{2}\right]^{1 / 3}$. We can show in the present study that a similar expression also holds when accounting for the magnetic field dependence of $m$ and $m_{\pi}$. Moreover, we have already proved analytically that $\mu_{B}^{\mathrm{BEC}}=m_{\pi}(B)$. Using this relation, the properly extended version of Eq. (40) given in Ref. [7], expressing both the effective quark mass and pion mass as functions of the magnetic field,

$$
\mu_{B}^{\mathrm{BEC} / \mathrm{BCS}}(B) \simeq\left[2 m(B) m_{\pi}^{2}(B)\right]^{1 / 3},
$$

turns out to be valid for all the values of the magnetic considered in the present work. In Fig. 8 we show our numerical results for $\mu_{B}^{\mathrm{BEC} / \mathrm{BCS}}(B)$ and the analytical expression obtained at finite $B$. It is clear that the result (4.8) holds in the present study.

The expression (4.8) is particularly useful to better understand the results of the previous section, shown in 
Fig. 4(b), as far as the behavior with the magnetic field is concerned. For small/intermediate values of $B$, the decrease of $\mu_{B}^{\mathrm{BEC}}(B)$ is basically compensated by the increase of the effective quark mass that follows from the MC effect. At around $e B=10 m_{\pi}^{2}$, both $m_{\pi}(B)$ and $m(B)$ display a sharp increase as a function of the magnetic field, leading to the observed behavior of $\mu_{B}^{\mathrm{BEC} / \mathrm{BCS}}(B)$.

\section{CONCLUSIONS}

In this work we studied the effect of the application of an external magnetic field on the BEC-BCS crossover. The model considered was the two-color NJL with diquark interactions. When including magnetic fields, special attention has to be paid to the regularization scheme used. We have considered in our studies three forms of regularization: one with the Lorentzian form factor (Lor5), another one using a Wood-Saxon form factor (WS0.05) and a recently studied regularization procedure (MFIR) in which the contributions that are explicitly dependent on the magnetic field are finite and, thus, do not have to be regularized. As shown in Ref. [34] in the two-flavor NJL model and also here when a diquark interaction term is included, the MFIR scheme fully prevents unphysical oscillatory behavior from appearing in the physical quantities, like in the condensates and in the critical chemical potentials.

Our results show that there is an IMC effect in the critical chemical potential for the BEC transition for diquark condensation. This is clearly observed for magnetic fields in the range $1 \lesssim e B / m_{\pi}^{2} \lesssim 20$. The critical chemical potential decreases during the IMC by at most $15 \%$, favoring the $\mathrm{BEC}$ transition. For larger values of the magnetic field, only magnetic catalysis is observed, requiring larger chemical potentials for the diquark condensation. With the further increase of the chemical potential, the system reaches the BEC-BCS crossover. The corresponding critical chemical potential is very weakly affected by the magnetic field up to values $e B \simeq 9 m_{\pi}^{2}$. There is still an IMC happening also for the crossover, but the phenomenon is much weaker than the one seen in the BEC transition with diquark condensation. The critical chemical potential for the crossover decreases at most by only $1.5 \%$. For larger magnetic fields, $e B \gtrsim 9 m_{\pi}^{2}$, the BEC-BCS crossover does however exhibit a magnetic catalysis effect. Thus, strong magnetic fields tend to strengthen the $\mathrm{BEC}$ region, once the difference $\mu_{B} / 2-m$ becomes positive at a higher value of $\mu_{B}$.

\section{ACKNOWLEDGMENTS}

D. C. D. and P. H. A. M. are supported by Coordenação de Aperfeiçoamento de Pessoal de Nível Superior (CAPES). R. L.S.F. and R. O. R are partially supported by research grants from Conselho Nacional de Desenvolvimento Científico e Tecnológico (CNPq). R. O. R. is also partially supported by a research grant from Fundação Carlos Chagas Filho de Amparo à Pesquisa do Estado do Rio de Janeiro (FAPERJ). P. G. A. and N. N. S. are partially supported by CONICET (Argentina) under grant PIP 00682 and by ANPCyT (Argentina) under grant PICT2011-0113. R. L. S. F. acknowledges the kind hospitality of the Center for Nuclear Research at Kent State University, where part of this work has been done. R. L.S.F. is also grateful to G. Endrödi for useful correspondence and comments.

\section{APPENDIX: THE MFIR SCHEME}

Let us briefly describe the MFIR scheme applied to the thermodynamic potential (2.5). We follow closely the procedure shown in Ref. [34], where the interested reader can find more details.

Let us consider the contribution of each flavor to the second term in Eq (2.5). It reads

$$
I_{f}=\frac{\left|q_{f}\right| B}{2 \pi} \sum_{s= \pm 1} \sum_{l=0}^{\infty} \alpha_{l} \int_{-\infty}^{+\infty} \frac{d k_{3}}{2 \pi} \sqrt{\left(E_{k_{3}, l}+s \mu\right)^{2}+\Delta^{2}} .
$$

Summing and subtracting a similar expression but where $\mu$ is set to zero, we get

$$
\begin{aligned}
I_{f}= & \frac{\left|q_{f}\right| B}{2 \pi} \sum_{l=0}^{\infty} \alpha_{l} \int_{-\infty}^{+\infty} \frac{d k_{3}}{2 \pi} F\left(k_{3}^{2}+2 l\left|q_{f}\right| B\right) \\
& +\frac{\left|q_{f}\right| B}{\pi} \sum_{l=0}^{\infty} \alpha_{l} \int_{-\infty}^{+\infty} \frac{d k_{3}}{(2 \pi)} \sqrt{E_{k_{3}, l}^{2}+\Delta^{2}}
\end{aligned}
$$

where $F\left(z^{2}\right)$ was defined in Eq. (2.10).

By adding and subtracting its form at vanishing magnetic field, the first term on the right-hand side of Eq. (A2) results in

$$
\begin{aligned}
\frac{\left|q_{f}\right| B}{2 \pi} & \sum_{l=0}^{\infty} \alpha_{l} \int_{-\infty}^{+\infty} \frac{d k_{3}}{2 \pi} F\left(k_{3}^{2}+2 l\left|q_{f}\right| B\right) \\
= & 2 \int \frac{d^{3} k}{(2 \pi)^{3}} F\left(\vec{k}^{2}\right)+\frac{\left|q_{f}\right| B}{(2 \pi)} \int_{-\infty}^{+\infty} \frac{d k_{3}}{(2 \pi)} F\left(k_{3}^{2}\right) \\
& +\frac{2\left|q_{f}\right| B}{2 \pi} \int_{-\infty}^{+\infty} \frac{d k_{3}}{2 \pi}\left\{\sum_{l=1}^{\infty} F\left(k_{3}^{2}+2 l\left|q_{f}\right| B\right)\right. \\
& \left.-\int_{0}^{\infty} d y F\left(k_{3}^{2}+2 y\left|q_{f}\right| B\right)\right\},
\end{aligned}
$$

where in the last term of the above expression we have used cylindrical coordinates, $\vec{k} \equiv\left(k_{\rho}, k_{\theta}, k_{3}\right)$, performed the angular integration and defined the new variable $y=k_{\rho}^{2} /\left(2\left|q_{f}\right| B\right)$. 
The second term on the right-hand side of Eq. (A2) can be evaluated using the method discussed in Ref. [26]. We get

$$
\begin{aligned}
& \frac{\left|q_{f}\right| B}{\pi} \sum_{l=0}^{\infty} \alpha_{l} \int_{-\infty}^{+\infty} \frac{d k_{3}}{(2 \pi)} \sqrt{E_{k_{3}, l}^{2}+\Delta^{2}} \\
& =4 \int \frac{d^{3} k}{(2 \pi)^{3}} \sqrt{k^{2}+m^{2}+\Delta^{2}} \\
& \quad+\frac{\left(\left|q_{f}\right| B\right)^{2}}{\pi^{2}}\left\{\zeta^{\prime}\left(-1, x_{f}\right)-\frac{1}{2}\left(x_{f}^{2}-x_{f}\right) \ln \left(x_{f}\right)+\frac{x_{f}^{2}}{4}\right\},
\end{aligned}
$$

where $x_{f}=\left(m^{2}+\Delta^{2}\right) /\left(2\left|q_{f}\right| B\right)$ and $\zeta^{\prime}(s, a)$ is the $s$ derivative of the Hurwitz zeta function [46].

Replacing Eqs. (A3) and (A4) in Eq. (A2) and using the definition of $F\left(z^{2}\right)$, Eq. (2.10), we get

$$
\begin{aligned}
I_{f}= & 2 \sum_{s= \pm 1} \int \frac{d^{3} k}{(2 \pi)^{3}} \sqrt{\left(E_{k}+s \mu\right)^{2}+\Delta^{2}} \\
& +\frac{\left(\left|q_{f}\right| B\right)^{2}}{\pi^{2}}\left\{\zeta^{\prime}\left(-1, x_{f}\right)-\frac{1}{2}\left(x_{f}^{2}-x_{f}\right) \ln \left(x_{f}\right)+\frac{x_{f}^{2}}{4}\right\} \\
& +\frac{\left|q_{f}\right| B}{2 \pi^{2}} \int_{0}^{\infty} d k_{3}\left\{\sum_{l=0}^{\infty} \alpha_{l} F\left(k_{3}^{2}+2 l\left|q_{f}\right| B\right)\right. \\
& \left.-2 \int_{0}^{\infty} d y F\left(k_{3}^{2}+2 y\left|q_{f}\right| B\right)\right\} .
\end{aligned}
$$

The first term in Eq. (A5) is exactly the $B=0$ contribution. It is divergent, but it can now be handled through standard methods, e.g., using a three-dimensional cutoff. At the same time, all the remaining terms in Eq. (A5) depending on the magnetic field are finite. Summing over flavors and using Eq. (2.3) we get Eq. (2.9).
[1] F. Karsch, Lect. Notes Phys. 583, 209 (2002).

[2] S. Muroya, A. Nakamura, C. Nonaka, and T. Takaishi, Prog. Theor. Phys. 110, 615 (2003).

[3] M. G. Alford, A. Schmitt, K. Rajagopal, and T. Schäfer, Rev. Mod. Phys. 80, 1455 (2008).

[4] Y. Nishida and H. Abuki, Phys. Rev. D 72, 096004 (2005); B. Chatterjee, H. Mishra, and A. Mishra, Phys. Rev. D 79, 014003 (2009); H. Abuki and T. Brauner, Phys. Rev. D 78, 125010 (2008).

[5] U. Vogl and W. Weise, Prog. Part. Nucl. Phys. 27, 195 (1991); S. Klevansky, Rev. Mod. Phys. 64, 649 (1992); T. Hatsuda and T. Kunihiro, Phys. Rep. 247, 221 (1994).

[6] M. Buballa, Phys. Rep. 407, 205 (2005).

[7] G. F. Sun, L. He, and P. Zhuang, Phys. Rev. D 75, 096004 (2007).

[8] M. Kitazawa, D. H. Rischke, and I. A. Shovkovy, Phys. Lett. B 663, 228 (2008).

[9] H. Abuki, G. Baym, T. Hatsuda, and N. Yamamoto, Phys. Rev. D 81, 125010 (2010).

[10] H. Basler and M. Buballa, Phys. Rev. D 82, 094004 (2010).

[11] E. J. Ferrer, V. de la Incera, J. P. Keith, and I. Portillo, Nucl. Phys. A933, 229 (2014).

[12] C. Ratti and W. Weise, Phys. Rev. D 70, 054013 (2004).

[13] T. Brauner, K. Fukushima, and Y. Hidaka, Phys. Rev. D 80, 074035 (2009); 81, 119904(E) (2010).

[14] L. He, Phys. Rev. D 82, 096003 (2010).

[15] L. He, S. Mao, and P. Zhuang, Int. J. Mod. Phys. A 28, 1330054 (2013).

[16] J. B. Kogut, D. K. Sinclair, S. J. Hands, and S. E. Morrison, Phys. Rev. D 64, 094505 (2001); S. Hands, I. Montvay, L. Scorzato, and J. Skullerud, Eur. Phys. J. C 22, 451 (2001).

[17] P. V. Buividovich, M. N. Chernodub, E. V. Luschevskaya, and M. I. Polikarpov, Phys. Lett. B 682, 484 (2010); Nucl.
Phys. B826, 313 (2010); P. V. Buividovich and M. I. Polikarpov, Phys. Rev. D 83, 094508 (2011).

[18] E.-M. Ilgenfritz, M. Kalinowski, M. Muller-Preussker, B. Petersson, and A. Schreiber, Phys. Rev. D 85, 114504 (2012).

[19] R. C. Duncan and C. Thompson, Astrophys. J. 392, L9 (1992); C. Kouveliotou et al., Nature (London) 393, 235 (1998).

[20] D. Lai and S. L. Shapiro, Astrophys. J. 383, 745 (1991).

[21] D. Bandyopadhyay, S. Chakrabarty, and S. Pal, Phys. Rev. Lett. 79, 2176 (1997).

[22] E. J. Ferrer, V. de la Incera, J. P. Keith, I. Portillo, and P. L. Springsteen, Phys. Rev. C 82, 065802 (2010).

[23] J. C. Wang, V. de la Incera, E. J. Ferrer, and Q. Wang, Phys. Rev. D 84, 065014 (2011).

[24] J. B. Kogut, M. A. Stephanov, D. Toublan, J. J. M. Verbaarschot, and A. Zhitnitsky, Nucl. Phys. B582, 477 (2000).

[25] A. Amador and J. O. Andersen, Phys. Rev. D 88, 025016 (2013).

[26] D. P. Menezes, M. B. Pinto, S. S. Avancini, A. P. Martínez, and C. Providência, Phys. Rev. C 79, 035807 (2009).

[27] J. L. Noronha and I. A. Shovkovy, Phys. Rev. D 76, 105030 (2007); 86, 049901(E) (2012).

[28] K. Fukushima and H. J. Warringa, Phys. Rev. Lett. 100, 032007 (2008).

[29] S. Fayazbakhsh and N. Sadooghi, Phys. Rev. D 82, 045010 (2010).

[30] T. Mandal and P. Jaikumar, Phys. Rev. C 87, 045208 (2013).

[31] L. Campanelli and M. Ruggieri, Phys. Rev. D 80, 034014 (2009).

[32] M. Frasca and M. Ruggieri, Phys. Rev. D 83, 094024 (2011).

[33] R. Gatto and M. Ruggieri, Lect. Notes Phys. 871, 87 (2013). 
[34] P. G. Allen, A. G. Grunfeld, and N. N. Scoccola, Phys. Rev. D 92, 074041 (2015).

[35] I. A. Shovkovy, Lect. Notes Phys. 871, 13 (2013).

[36] V. Skokov, A. Illarionov, and V. Toneev, Int. J. Mod. Phys. A 24, 5925 (2009).

[37] D. Ebert, K. G. Klimenko, M. A. Vdovichenko, and A. S. Vshivtsev, Phys. Rev. D 61, 025005 (1999).

[38] T. Inagaki, D. Kimura, and T. Murata, Prog. Theor. Phys. 111, 371 (2004).

[39] F. Preis, A. Rebhan, and A. Schmitt, Lect. Notes Phys. 871, 51 (2013); J. High Energy Phys. 03 (2011) 033.

[40] J. L. Kneur, M. B. Pinto, and R. O. Ramos, Phys. Rev. D 88, 045005 (2013).

[41] S. S. Avancini, W. R. Tavares, and M. B. Pinto, arXiv:1511.06261 [Phys. Rev. D (to be published)].

[42] G. Endrödi, Phys. Rev. D 90, 094501 (2014).
[43] N. Strodthoff, B. J. Schaefer, and L. von Smekal, Phys. Rev. D 85, 074007 (2012).

[44] L. He, M. Jin, and P. Zhuang, Phys. Rev. D 71, 116001 (2005).

[45] D. T. Son and M. A. Stephanov, Phys. Rev. Lett. 86, 592 (2001); Phys. At. Nucl. 64, 834 (2001); J. B. Kogut, M. A. Stephanov, and D. Toublan, Phys. Lett. B 464, 183 (1999); J. T. Lenaghan, F. Sannino, and K. Splittorff, Phys. Rev. D 65, 054002 (2002); K. Splittorff, D. Toublan, and J. J. M. Verbaarschot, Nucl. Phys. B620, 290 (2002); B639, 524 (2002); L. von Smekal, Nucl. Phys. B, Proc. Suppl. 228, 179 (2012); K. Splittorff, D. T. Son, and M. A. Stephanov, Phys. Rev. D 64, 016003 (2001); J. O. Andersen and T. Brauner, Phys. Rev. D 81, 096004 (2010).

[46] E. Elizalde, A. D. Odintsov, and A. Romeo, Zeta Regularization Techniques with Applications, (World Scientific, Singapore, 1994). 